\title{
Matching the non-equilibrium initial stage of heavy ion collisions to hydrodynamics with QCD kinetic theory
}

\author{
Aleksi Kurkela \\ Theoretical Physics Department, CERN, Geneva, Switzerland \\ Faculty of Science and Technology, University of Stavanger, 4036 Stavanger, Norway \\ E-mail: a.kecern.ch
}

Aleksas Mazeliauskas*

Institut für Theoretische Physik, Universität Heidelberg, Heidelberg 69120, Germany

E-mail: a.mazeliauskas@thphys.uni-heidelberg.de

\section{Jean-François Paquet}

Department of Physics, Duke University, Durham, NC 27708, USA

E-mail: jeanfrancois.paquet@duke.edu

\section{Sören Schlichting}

Fakultät für Physik, Universität Bielefeld, D-33615 Bielefeld, Germany

E-mail: sschlichting@physik.uni-bielefeld.de

\section{Derek Teaney}

Department of Physics and Astronomy, Stony Brook University, Stony Brook, NY 11794, USA

E-mail: derek.teaneyestonybrook. edu

\begin{abstract}
In the collision of nuclei at high energies the produced matter reinteracts and forms a plasma which ultimately equilibrates and exhibits collective hydrodynamic flow. While a general theory of the equilibration process has been outlined previously, there were no practical frameworks to smoothly connect the early gluon production in classical field simulations with hydrodynamic simulations of the late time plasma expansion. We provide this practical tool (called KøMPøST) by constructing a set of non-equilibrium Green functions calculated in QCD kinetic theory. We demonstrate with a realistic simulation of a heavy ion collisions the smooth transition from the classical fields to hydrodynamics, and calculate the pragmatic lower bound on the system size for the hydrodynamics to be applicable.
\end{abstract}

XIII Quark Confinement and the Hadron Spectrum - Confinement2018

31 July - 6 August 2018

Maynooth University, Ireland

${ }^{*}$ Speaker. 


\section{Introduction}

In the last couple of decades there has been an intense experimental and theoretical effort in studying the deconfined state of nuclear matter-the quark-gluon plasma (QGP) - which is believed to be formed in relativistic nucleus-nucleus collisions. A surprisingly successful description of the plasma expansion is given by relativistic viscous hydrodynamics [1, 2, 3]. Model to data comparisons allow to constrain fundamental properties of QCD medium, e.g shear viscosity over entropy ratio $\eta / s$ or the QCD equation of state. However, the study of how such macroscopic medium description can arise from the microscopic far-from-equilibrium QCD physics is an active field of research $[4,5,6]$.

A significant progress in describing the initial stages of heavy ion collisions from first principles can be made in the high-energy limit of centre of mass energy $\sqrt{s} \rightarrow \infty$, where weakly coupled effective descriptions of QCD are applicable. In the color glass condensate (CGC) framework the far-from-equilibrium initial state is dominated by highly occupied gluonic degrees of freedom, which are evolved by classical-statistical Yang-Mills equations of motion [7, 8, 9, 10, 11]. However classical fields never thermalize and once the system is sufficiently dilute, the quantum corrections can no longer be neglected $[12,13,14]$. At this point an alternative descriptions in terms of kinetic theory of quasi-particles is more appropriate [15]. The path from CGC initial conditions to equilibrium has been laid down in the seminal "bottom-up" thermalization paper [16]. Detailed classicalstatistical evolution of gluon fields show that the evolution of bulk quantities indeed follow the first stage of bottom-up thermalization picture [17, 18, 19]. In later stages (described by kinetic theory) particles scatter to isotropize the expanding plasma and bring the system towards thermal and hydrodynamic equilibrium. Such approach to equilibrium for homogeneous expanding systems was realized numerically in recent kinetic simulations [20]. However, realistic initial conditions of heavy-ion collisions have non-trivial transverse geometry. In this work [21, 22], we apply the weakly coupled QCD kinetic theory to propagated transverse perturbations in out-of-equilibrium plasma and provide a framework (called KøMPøST) to do this evolution in event-by-event nucleusnucleus collisions [23].

\section{Equilibration and hydrodynamization in QCD kinetic theory}

The finite temperature QCD processes are highly non-trivial and require resummation of thermal loop integrals even at leading order, which generate effective temperature dependent screening masses of quark and gluon quasi-particles [24]. The evolution of such quasi-particles is then given by the following Boltzmann equation [15]

$$
\partial_{\tau} f+\frac{\mathbf{p}}{|p|} \cdot \nabla f-\frac{p_{z}}{\tau} \partial_{p_{z}} f=-\mathscr{C}_{2 \leftrightarrow 2}[f]-\mathscr{C}_{1 \leftrightarrow 2}[f]
$$

where $f(\mathbf{p}, \mathbf{x}, \tau)$ is the particle phase-space distribution and $\mathscr{C}_{2 \leftrightarrow 2}[f]$ is the leading order collision integral for elastic $2 \leftrightarrow 2$ scatterings. For the soft small angle scattering the divergent tree level scattering matrix is regulated by the screening masses [25]. At the same order of the coupling, interactions with the soft gluonic field fluctuations induces number changing processes, which are denoted by the effective $1 \leftrightarrow 2$ process $\mathscr{C}_{1 \leftrightarrow 2}[f]$ and include the Landau-Pomeranchuk-Migdal 

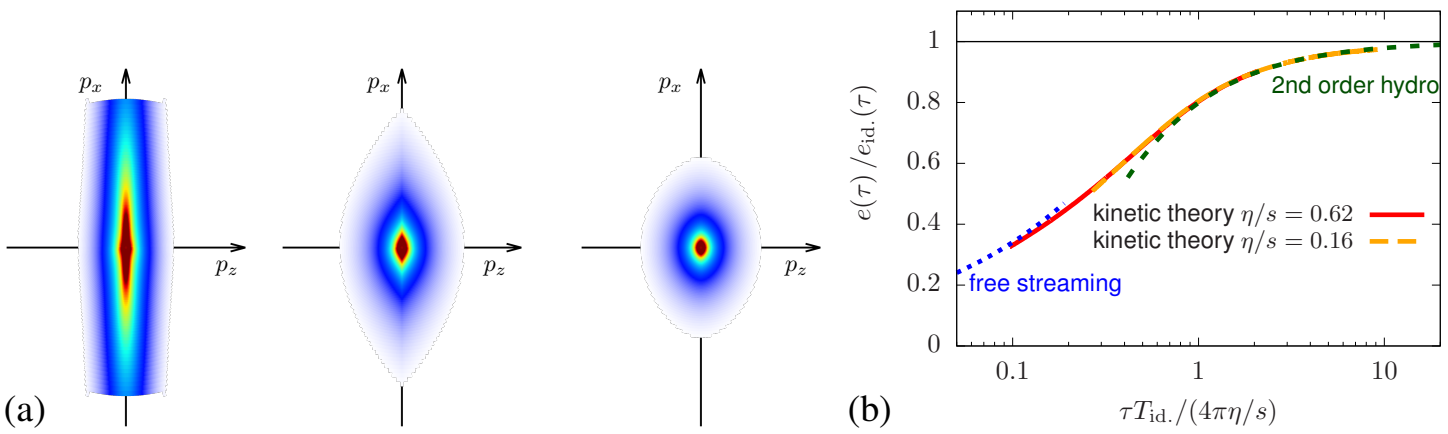

Figure 1: (a) The gluon phase-space distribution at different stages of kinetic pre-equilibrium evolution at $\tau T_{\text {id. }} /(4 \pi \eta / s) \approx 0.1,0.5,1.0$. Figure adapted from [21] (b) The energy density evolution in kinetic theory for different values of the coupling constant (or $\eta / s$ ). Figure adapted from [22].

suppression of collinear radiation. This description is leading order accurate in the QCD coupling constant $\lambda=N_{c} g^{2}$ - the only model parameter-for out-of-equilibrium, but parametrically isotropic weakly coupled plasma [15]. Realistic conditions in heavy ion collisions are probably different from this idealized limit, but we may still use this first principle description of QGP to understand the equilibration processes [26, 20, 27, 28]. In the following we use the QCD kinetic theory only with gluon scattering processes included - the equilibration using the full QCD degrees of freedom was recently studied in $[29,30]$.

First starting from the CGC inspired initial gluon distribution we numerically solve the Boltzmann equation Eq. (2.1) for homogeneous boost invariant system [20]. Different stages of the phase-space distribution evolution is shown in Fig. 1(a). The elastic scattering process tend isotropize the distribution, while collinear cascade transfers energy from high to low momentum. It is notable that during this isotropization process a large amount of entropy is generated and the gluon number increases by a factor of $\sim 2$ [21]. In Fig. 1(b) the time evolution of the first moment of the same distribution, i.e. the energy density

$$
e(\tau)=v_{g} \int \frac{d^{3} p}{(2 \pi)^{3}} p^{0} f(p, \tau)
$$

is shown $\left(v_{g}=2\left(N_{c}^{2}-1\right)\right)$. We observe that the late time behavior of energy density is well described by viscous hydrodynamics, which for the homogeneous boost invariant expansion can be written as a gradient expansion

$$
e(\tau)=v_{g} \frac{\pi^{2}}{30} T_{\text {Id. }}^{4}\left(1-\frac{8}{3} \frac{\eta / s}{\tau T_{\text {Id. }}}+C_{2}\left(\frac{\eta / s}{\tau T_{\text {Id. }}}\right)^{2}\right) .
$$

Here $T_{\text {id. }}=\left(\tau^{1 / 3} T\right)_{\infty} / \tau^{1 / 3}$ is the 'ideal' temperature defined by the asymptotic constant $\left(\tau^{1 / 3} T\right)_{\infty}=$ $\lim _{\tau \rightarrow \infty} \tau^{1 / 3} T$. However, we find that even at earlier times the energy density, which is no longer described just by the gradient expansion Eq. (2.3), still is a single variable function of the scaled time $\tau / \tau_{R}$, where $\tau_{R}$ is the kinetic relaxation time $\tau_{R}=\eta / s T_{\text {id. }}$. This can be seen from a good overlap of kinetic evolution with different coupling constants (and effective $\eta / s$ ) shown in Fig. 1(b). After the rescaling of the axes both kinetic evolutions coincide. For the homogeneous conformal 


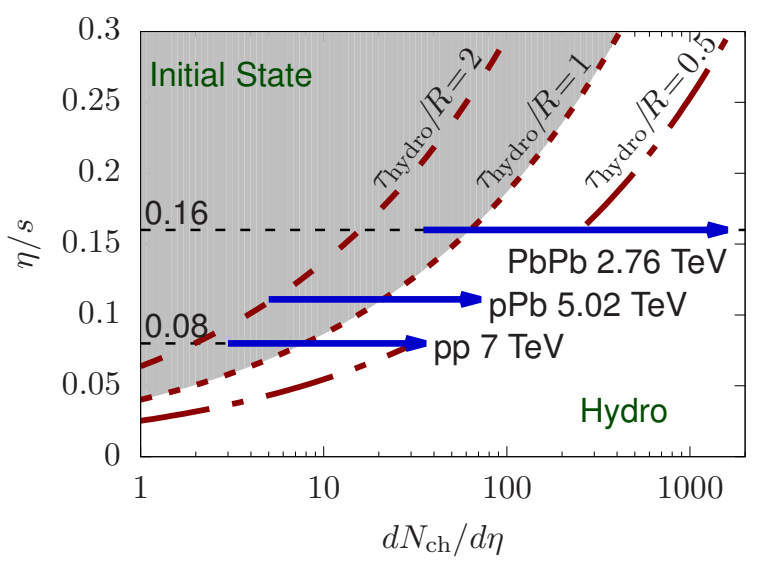

Figure 2: Contour plot of hydrodynamization time to system size ratio $\tau_{\text {hydro }} / R$ as a function specific shear viscosity $\eta / s$ and charged particle multiplicity $d N_{\mathrm{ch}} / d \eta$. Figure adapted from [31].

system energy density is the only independent component of the energy-momentum tensor, and therefore the universal curve in Fig. 1(b) fully characterises the early time dynamics of $T^{\mu v}$ of such system.

The universality pre-equilibrium evolution in rescaled units enables us to determine the relation between the system size and the equilibration time. As is seen from Fig. 1(b) hydrodynamics is a good description at $\tau_{\text {hydro }} \approx 4 \pi \tau_{R}$. Note that the system can be considered undergoing a onedimensional expansion only for times smaller than the transverse radius of the system $\tau<R$ [22]. Since a three-dimensional expansion is more rapid, it is unlikely that a system will equilibrated if $\tau_{\text {hydro }}>R$. Therefore the requirement that $\tau_{\text {hydro }} / R<1$ can be used as a bound for minimal particle multiplicity $d N_{\mathrm{ch}} / d \eta$ for a system to reach hydrodynamization before falling apart. Relating the entropy density in the system $d S / d y \propto d N_{\mathrm{ch}} / d \eta / A_{\perp}$, where $A_{\perp}=\pi R^{2}$ is the transverse area of the system, to the asymptotic constant $\left(\tau^{1 / 3} T\right)_{\infty}$ we arrive at the following equation [22]

$$
\frac{\tau_{\mathrm{hydro}}}{R} \approx\left(\frac{4 \pi(\eta / s)}{2}\right)^{\frac{3}{2}}\left(\frac{d N_{\mathrm{ch}} / d \eta}{63}\right)^{-\frac{1}{2}} .
$$

We plotted Eq. (2.4) as a $\tau_{\text {hydro }} / R$ contour plot in Fig. 2 and indicated typical multiplicity ranges for $\mathrm{p}-\mathrm{p}, \mathrm{p}-\mathrm{Pb}$ and $\mathrm{Pb}-\mathrm{Pb}$ collisions on the plot. Although the ratio $\tau_{\text {hydro }} / R$ is strongly dependent on the specific shear viscosity, the multiplicity in nucleus-nucleus collisions can be sufficiently large for the plasma to hydrodynamize (i.e. $\tau_{\text {hydro }} \ll R$ ) for a range of physical values of $\eta / s$. However in smaller collisions systems with multiplicities $d N_{\mathrm{ch}} / d \eta<10$ hydrodynamization is unlikely as $\tau_{\text {hydro }}>R$ even for a very small value $\eta / s \approx 0.08$. Therefore Eq. (2.4) can be viewed as a pragmatic lower bound on the system size when hydrodynamics becomes applicable.

\section{Transverse pre-equilibrium dynamics}

Realistic heavy ion events have non-trivial transverse geometry and kinetic theory pre-equilibrium requires evolution of transverse perturbations. While complete space-time evolution in kinetic theory is computationally expensive, a practical pre-equilibrium evolution can be given by linear 


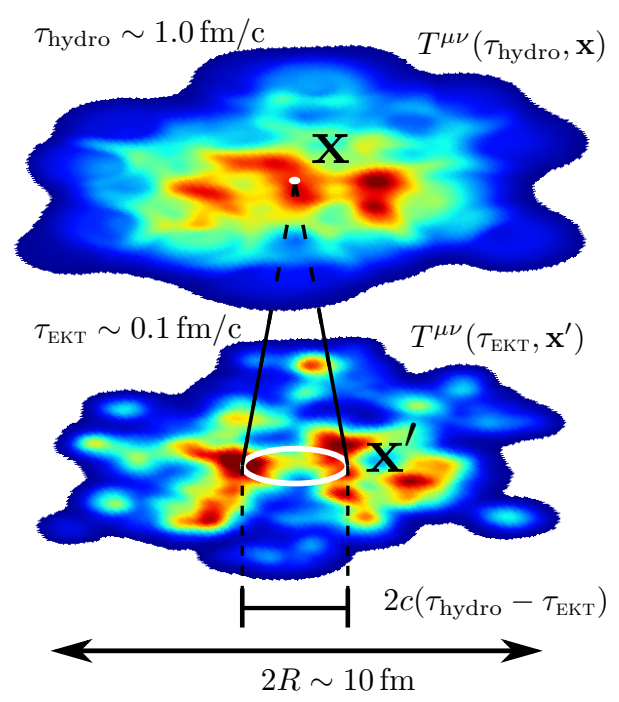

Figure 3: Transverse energy density profile before and after kinetic pre-equilibrium evolution. White circle indicates the size of causal circle. Figure adapted from [22]

non-equilibrium response functions [28, 21, 22]. For large collision systems and short evolution times we can use the separation of scales between the system size and the causal circle of the pre-equilibrium evolution shown in Fig. 3

$$
R \gg c\left|\tau_{\text {hydro }}-\tau_{\text {EKT }}\right| .
$$

The energy-momentum tensor can be decomposed locally in a homogeneous background and (small) transverse perturbations

$$
T^{\mu v}\left(\tau_{\mathrm{EKT}}, \mathbf{x}^{\prime}\right)=\bar{T}_{\mathbf{x}}^{\mu v}\left(\tau_{\mathrm{EKT}}\right)+\delta T_{\mathbf{x}}^{\mu v}\left(\tau_{\mathrm{EKT}}, \mathbf{x}^{\prime}\right)
$$

Then the equilibration proceeds in two stages. The uniform, but highly anisotropic background energy-momentum tensor $\bar{T}^{\mu v}$ equilibrates according to the scaling curve Fig. 1(b) as described in the previous section. Evolution of phase-space distribution perturbations corresponding to $\delta T^{\mu v}$ is then given by the linearized Boltzmann equation around the background solution [28]. As we are interested in the late time behavior of the energy-momentum tensor, it is convenient to extract just the energy-momentum tensor evolution from the simulation of the full phase-space distribution. Using the results of explicit kinetic theory evolution we construct non-equilibrium response functions $G_{\alpha \beta}^{\mu v}\left(\mathbf{x}, \mathbf{x}_{0}, \tau, \tau_{0}\right)$ which propagate perturbations associated with initial energy $\left(\delta T^{\tau \tau}\right)$ and momentum $\left(\delta T^{\tau i}\right)$ perturbations

$$
\frac{\delta T^{\mu \nu}(\tau, \mathbf{x})}{\bar{T}_{\mathbf{x}}^{\tau \tau}(\tau)}=\frac{1}{\bar{T}_{\mathbf{x}}^{\tau \tau}\left(\tau_{0}\right)} \int d^{2} \mathbf{x}_{0} G_{\alpha \beta}^{\mu \nu}\left(\mathbf{x}, \mathbf{x}_{0}, \tau, \tau_{0}\right) \delta T_{\mathbf{x}}^{\alpha \beta}\left(\tau_{0}, \mathbf{x}_{0}\right) .
$$

In general, even a single phase-space perturbation corresponding to energy perturbation in the initial conditions will generate contributions to all components of $T^{\mu v}$. Therefore multiple response functions are needed. Using rotational symmetry in the transverse plane, one can decompose the response functions into a tensor basis [21]. For energy perturbations the coordinate space response 

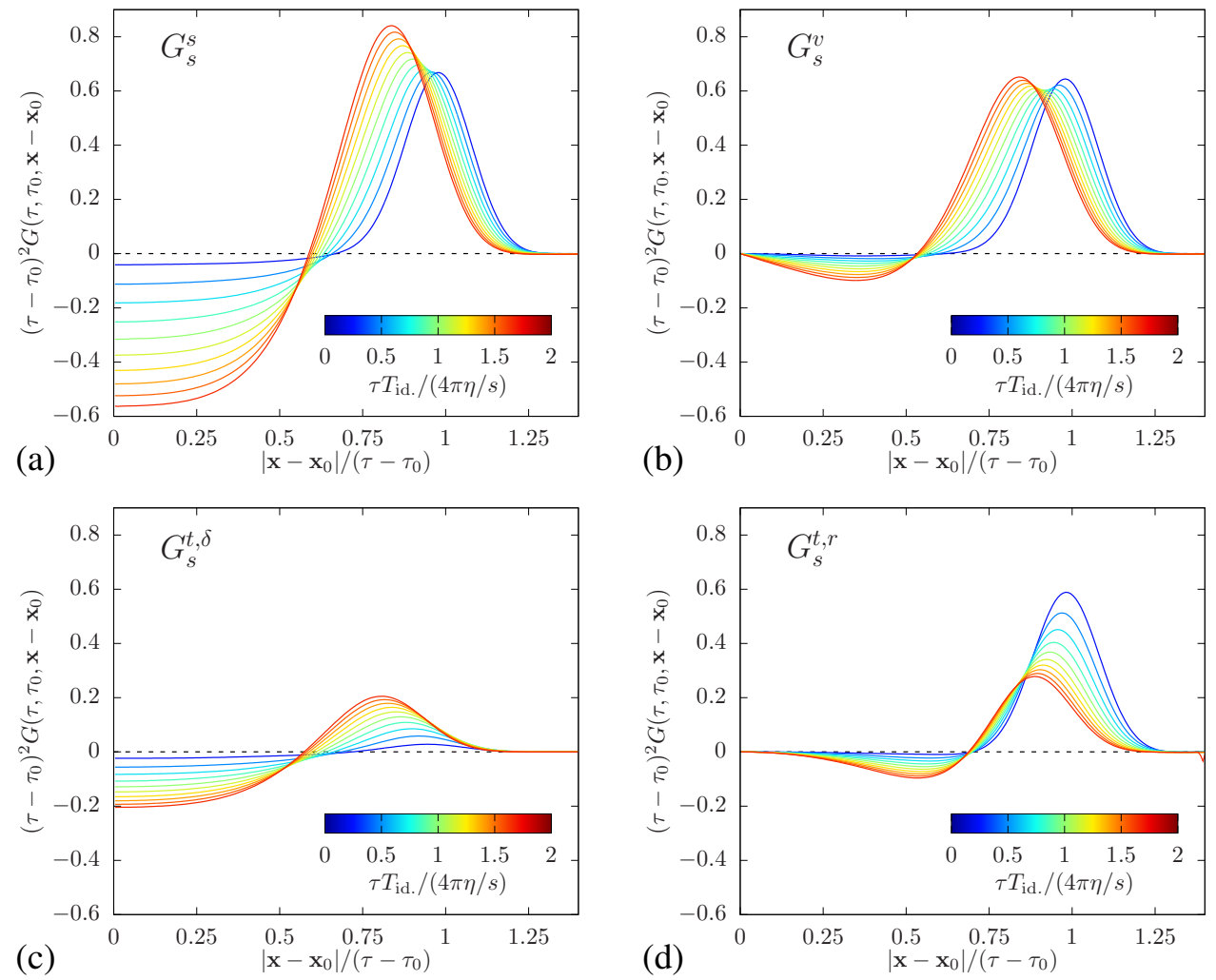

Figure 4: Independent components of energy-momentum tensor response to initial energy perturbation. Figure adapted from [21]

function $G_{\tau \tau}^{\mu v}\left(\tau, \tau_{0}, \mathbf{r}=\mathbf{x}-\mathbf{x}_{0}\right)$ has four independent structures

$$
\begin{aligned}
G_{s}^{\tau \tau}\left(\tau, \tau_{0}, \mathbf{r}\right) & =G_{s}^{s}\left(\tau, \tau_{0},|\mathbf{r}|\right), \quad G_{s}^{\tau i}\left(\tau, \tau_{0}, \mathbf{r}\right)=\frac{\mathbf{r}^{i}}{|\mathbf{r}|} G_{s}^{v}\left(\tau, \tau_{0},|\mathbf{r}|\right), \\
G_{s}^{i j}\left(\tau, \tau_{0}, \mathbf{r}\right) & =G_{s}^{t, \delta}\left(\tau, \tau_{0},|\mathbf{r}|\right) \delta^{i j}+G_{s}^{t, r}\left(\tau, \tau_{0},|\mathbf{r}|\right) \frac{\mathbf{r}^{i} \mathbf{r}^{j}}{|\mathbf{r}|^{2}}
\end{aligned}
$$

which are shown in Fig. 4 at different evolution times in scaled variables. The response function smoothly transitions from free-streaming-like response $G \propto \delta(\Delta \tau-|\Delta \mathbf{x}|)$ to late time hydrodynamic response with effective signal propagation speed $c_{s}<1$. Note that close to equilibrium, the shear-stress response functions $G_{s}^{t, \delta}$ and $G_{s}^{t, r}$ are not independent, but satisfy hydrodynamic constituent equations [21]. This ensures a smooth overlap of kinetic and hydrodynamic descriptions for the perturbations at late times. Analogously to energy perturbations, one constructs response to initial momentum perturbations $\delta T^{\tau i}$, which have six independent response functions. Importantly the response functions depend on the specific shear viscosity or background energy not directly, but only through the scaled time variable $\frac{\tau T_{\mathrm{id}}}{\eta / s}$. This allows the reuse of the tabulated response functions for the propagation of initial perturbations for different background energy density and effective $\eta / s$. 

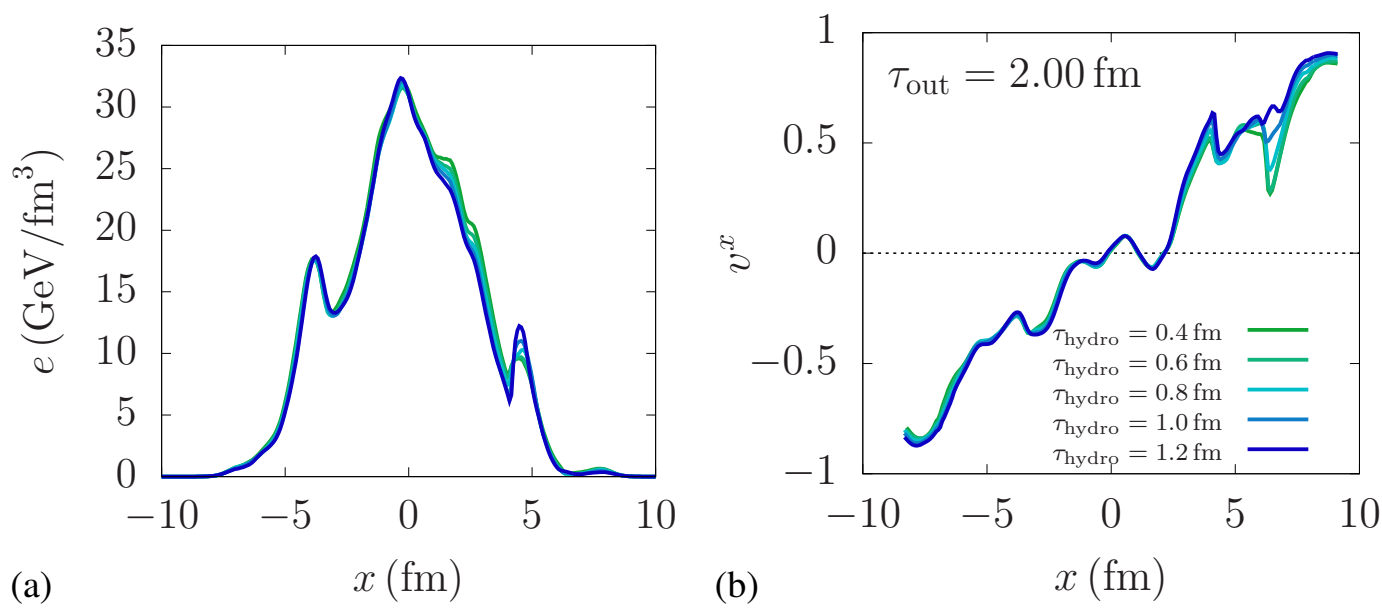

Figure 5: (a) Energy density and (b) transverse velocity in the hydrodynamic evolution with different KøMPøST evolution times. Figures adapted from [22].

\section{KøMPøST}

The tabulated evolution as a function of scaled variable $\tau / \tau_{R}$ of the background Fig. 1(b) and the response functions, e.g. Fig. 4, can be straightforwardly applied to the event-by-event equilibration of out-of-equilibrium initial conditions in heavy ion collisions. We constructed an implementation of such linearized kinetic pre-equilibrium propagator—called KøMPøST—which is publicly available [23].

The input for KøMPøST can be supplied by CGC based initial state model IP-Glasma [32, 33] or initial state parametrization models, e.g. TRENTo [34]. At the kinetic starting time $\tau_{\mathrm{EKT}} \sim 0.1 \mathrm{fm}$ the initial out-of-equilibrium energy momentum tensor $T^{\mu v}(\tau, \mathbf{x})$ is passed to KøMPøST. Then for each point in the transverse plane the decomposition into local background and perturbations is performed, Eq. (3.2). The decomposition is arbitrary as long as perturbations are small and we use Gaussian averaging with $2 \sigma=\left|\tau_{\text {hydro }}-\tau_{\text {ЕКT }}\right|$ to find the background energy density $\bar{T}^{\tau \tau}$. Then $\bar{T}^{\tau \tau}, \tau_{\mathrm{EKT}}$ and $\eta / s$ uniquely determines the position on the scaling curve Fig. 1(b), which is given by analytically parametrized function in the code [23, 21]. The final background energy density and pressures can be read off from the same scaling curve at some later time $\tau_{\text {hydro }}$. The energymomentum tensor components which deviate from the averaged homogeneous background $\bar{T}^{\mu v}$ are propagated as perturbations. In this work we evolve only initial energy $\left(\delta T^{\tau \tau}\right)$ and transverse momentum $\left(\delta T^{\tau i}\right)$ components. Shear-stress perturbations $\delta T^{i j}$ —which do not correspond to conserved charges - are neglected. The final energy-momentum tensor $T^{\mu v}\left(\mathbf{x}, \tau_{\text {hydro }}\right)$ is as a sum of the background $\bar{T}^{\mu v}\left(\mathbf{x}, \tau_{\text {hydro }}\right)$ and perturbations $\delta T^{\mu v}\left(\mathbf{x}, \tau_{\text {hydro }}\right)$ obtained from the convolution with the response functions in Eq. (3.3). Finally, the approximately equilibrated $T^{\mu v}$ can be passed to the subsequent hydrodynamic evolution.

The agreement of hydrodynamic and kinetic evolution, e.g. as seen in Fig. 1(b), ensures that the final observables are not sensitive to the exact switching time. To demonstrate this, we performed the pre-equilibrium evolution of IP-Glasma based initial conditions from $\tau_{\mathrm{EKT}}=0.2 \mathrm{fm}$ to $\tau_{\text {hydro }}=0.6-1.2 \mathrm{fm}$ and read out the the energy density and transverse velocity from the hydro- 

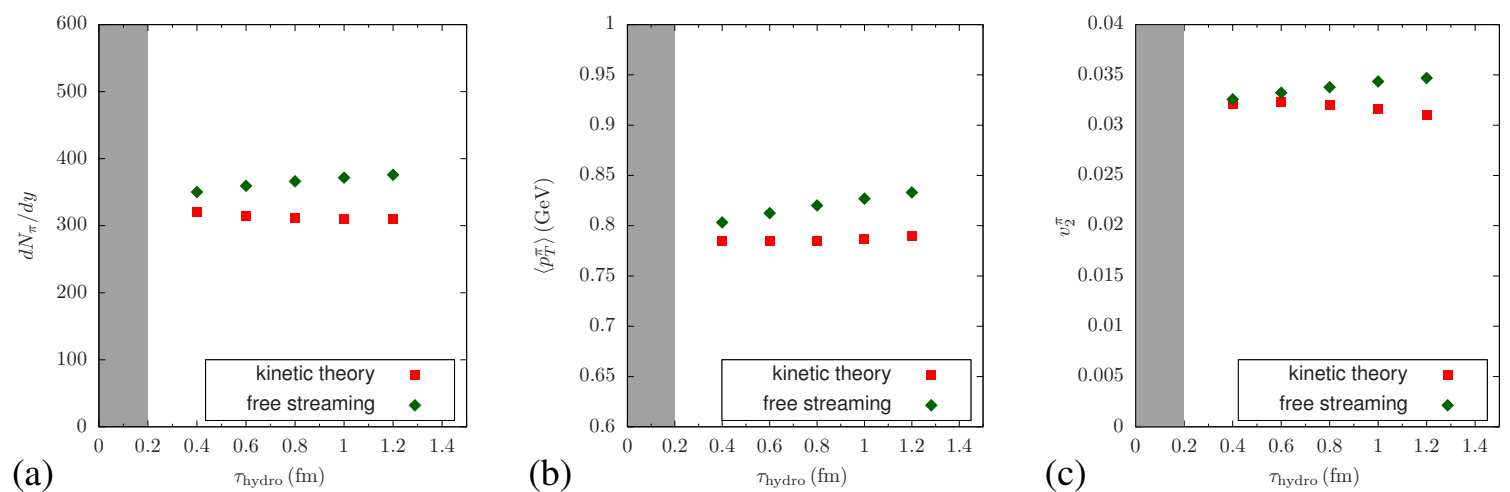

Figure 6: (a) Pion multiplicity (b) mean $p_{T}$ and (c) elliptic flow with varying duration of KøMPøST and free-streaming pre-equilibrium evolution. Figure adapted from [21]

dynamic evolution at a fixed time $\tau_{\text {out }}=2 \mathrm{fm}$. As shown in Fig. 5 the good overlap of curves demonstrate a smooth transition from kinetic to hydrodynamic evolution. Note that at the edges of the fireball-where gradients are especially large - the linearized description is failing and the local fluid rest-frame may not be found. In these isolated cases a regulator is used to facilitate the initialization of the subsequent hydrodynamic evolution [21].

Finally, at the end of the hydrodynamic evolution the hadronic observables, e.g. pion multiplicity $d N / d y$, mean $p_{T}$ and $v_{2}$ are calculated using the standard freeze-out procedures [21]. The dependence of hadronic observables on the switching time $\tau_{\text {hydro }}$ from KøMPøST to hydrodynamic evolution is shown in Fig. 6 by red points. Comparing with free-streaming pre-equilibrium shown by green points we see that KøMPøST pre-equilibrium is less sensitive to the switching time. In free streaming the energy density is falling slower than in hydrodynamic and therefore the final multiplicity increases with later hydro initialization times. To keep the final particle multiplicity fixed, one would normally readjust the energy density at each starting time. As seen from Fig. 6 no such readjustments are needed for KøMPøST pre-equilibrium. It is important to emphasize that KøMPøST outputs all components of $T^{\mu v}$, which smoothly and automatically approach the energy-momentum tensor of the hydrodynamically described medium. Therefore no additional assumptions about the transverse flow or pressures are needed to initialize the subsequent hydrodynamic evolution at $\tau_{\text {hydro }}$.

\section{Discussion}

We presented a practical implementation of pre-equilibrium evolution of initial conditions for heavy ion collisions based on the QCD kinetic theory. Using the numerical solutions of Boltzmann equation we described the evolution of out-of-equilibrium energy-momentum tensor for CGC motivated initial conditions in terms of kinetic response functions. The publicly available package KøMPøST [21] provides the pre-equilibrium description for event-by-event simulations, which can be straightforwardly incorporated in current hydrodynamic models of heavy ion collisions. We demonstrated that this framework smoothly matches to the hydrodynamic evolution and reduces the sensitivity to the hydrodynamic initialization time. Reducing the dependence on such ad-hoc 
model parameters could improve the extraction of transport properties of the QGP from model to data comparisons.

\section{Acknowledgments}

This work was supported in part by the German Research Foundation (DFG) Collaborative Research Centre (SFB) 1225 (ISOQUANT) (A.M.)

\section{References}

[1] U. Heinz and R. Snellings, Ann. Rev. Nucl. Part. Sci. 63, 123 (2013) doi:10.1146/annurev-nucl-102212-170540 [arXiv:1301.2826 [nucl-th]].

[2] C. Gale, S. Jeon and B. Schenke, Int. J. Mod. Phys. A 28, 1340011 (2013) doi:10.1142/S0217751X13400113 [arXiv:1301.5893 [nucl-th]].

[3] R. Derradi de Souza, T. Koide and T. Kodama, Prog. Part. Nucl. Phys. 86, 35 (2016) doi:10.1016/j.ppnp.2015.09.002 [arXiv:1506.03863 [nucl-th]].

[4] A. Kurkela, Nucl. Phys. A 956, 136 (2016) doi:10.1016/j.nuclphysa.2016.01.069 [arXiv:1601.03283 [hep-ph]].

[5] P. Romatschke, Phys. Rev. Lett. 120, no. 1, 012301 (2018) doi:10.1103/PhysRevLett.120.012301 [arXiv:1704.08699 [hep-th]].

[6] A. Kurkela, U. A. Wiedemann and B. Wu, arXiv:1805.04081 [hep-ph].

[7] T. Lappi and L. McLerran, Nucl. Phys. A 772, 200 (2006) doi:10.1016/j.nuclphysa.2006.04.001 [hep-ph/0602189].

[8] E. Iancu, A. Leonidov and L. McLerran, hep-ph/0202270.

[9] E. Iancu and R. Venugopalan, In *Hwa, R.C. (ed.) et al.: Quark gluon plasma* 249-3363 doi:10.1142/9789812795533_0005 [hep-ph/0303204].

[10] F. Gelis, E. Iancu, J. Jalilian-Marian and R. Venugopalan, Ann. Rev. Nucl. Part. Sci. 60, 463 (2010) doi:10.1146/annurev.nucl.010909.083629 [arXiv:1002.0333 [hep-ph]].

[11] F. Gelis, T. Lappi and R. Venugopalan, Int. J. Mod. Phys. E 16, 2595 (2007) doi:10.1142/S0218301307008331 [arXiv:0708.0047 [hep-ph]].

[12] G. Aarts and J. Berges, Phys. Rev. Lett. 88, 041603 (2002) doi:10.1103/PhysRevLett.88.041603 [hep-ph/0107129].

[13] A. H. Mueller and D. T. Son, Phys. Lett. B 582, 279 (2004) doi:10.1016/j.physletb.2003.12.047 [hep-ph/0212198].

[14] S. Jeon, Phys. Rev. C 72, 014907 (2005) doi:10.1103/PhysRevC.72.014907 [hep-ph/0412121].

[15] P. B. Arnold, G. D. Moore and L. G. Yaffe, JHEP 0301, 030 (2003) doi:10.1088/1126-6708/2003/01/030 [hep-ph/0209353].

[16] R. Baier, A. H. Mueller, D. Schiff and D. T. Son, Phys. Lett. B 502, 51 (2001) doi:10.1016/S0370-2693(01)00191-5 [hep-ph/0009237].

[17] J. Berges, K. Boguslavski, S. Schlichting and R. Venugopalan, Phys. Rev. D 89, no. 7, 074011 (2014) doi:10.1103/PhysRevD.89.074011 [arXiv:1303.5650 [hep-ph]]. 
[18] J. Berges, K. Boguslavski, S. Schlichting and R. Venugopalan, Phys. Rev. D 89, no. 11, 114007 (2014) doi:10.1103/PhysRevD.89.114007 [arXiv:1311.3005 [hep-ph]].

[19] J. Berges, B. Schenke, S. Schlichting and R. Venugopalan, Nucl. Phys. A 931, 348 (2014) doi:10.1016/j.nuclphysa.2014.08.103 [arXiv:1409.1638 [hep-ph]].

[20] A. Kurkela and Y. Zhu, Phys. Rev. Lett. 115, no. 18, 182301 (2015) doi:10.1103/PhysRevLett.115.182301 [arXiv:1506.06647 [hep-ph]].

[21] A. Kurkela, A. Mazeliauskas, J. F. Paquet, S. Schlichting and D. Teaney, Phys. Rev. C 99, no. 3, 034910 (2019) doi:10.1103/PhysRevC.99.034910 [arXiv:1805.00961 [hep-ph]].

[22] A. Kurkela, A. Mazeliauskas, J. F. Paquet, S. Schlichting and D. Teaney, Phys. Rev. Lett. 122, no. 12, 122302 (2019) doi:10.1103/PhysRevLett.122.122302 [arXiv:1805.01604 [hep-ph]].

[23] A. Kurkela, A. Mazeliauskas, J. F. Paquet, S. Schlichting and D. Teaney, https://github.com/KMPST/KoMPoST (2018).

[24] E. Braaten and R. D. Pisarski, Nucl. Phys. B 337, 569 (1990). doi:10.1016/0550-3213(90)90508-B

[25] M. C. Abraao York, A. Kurkela, E. Lu and G. D. Moore, Phys. Rev. D 89, no. 7, 074036 (2014) doi:10.1103/PhysRevD.89.074036 [arXiv:1401.3751 [hep-ph]].

[26] A. Kurkela and E. Lu, Phys. Rev. Lett. 113, no. 18, 182301 (2014) doi:10.1103/PhysRevLett.113.182301 [arXiv:1405.6318 [hep-ph]].

[27] L. Keegan, A. Kurkela, P. Romatschke, W. van der Schee and Y. Zhu, JHEP 1604, 031 (2016) doi:10.1007/JHEP04(2016)031 [arXiv:1512.05347 [hep-th]].

[28] L. Keegan, A. Kurkela, A. Mazeliauskas and D. Teaney, JHEP 1608, 171 (2016) doi:10.1007/JHEP08(2016)171 [arXiv:1605.04287 [hep-ph]].

[29] A. Kurkela and A. Mazeliauskas, Phys. Rev. D 99, no. 5, 054018 (2019) doi:10.1103/PhysRevD.99.054018 [arXiv:1811.03068 [hep-ph]].

[30] A. Kurkela and A. Mazeliauskas, Phys. Rev. Lett. 122, 142301 (2019) doi:10.1103/PhysRevLett.122.142301 [arXiv:1811.03040 [hep-ph]].

[31] A. Mazeliauskas, Nucl. Phys. A 982, 134 (2019) doi:10.1016/j.nuclphysa.2018.08.019 [arXiv:1807.05586 [nucl-th]].

[32] B. Schenke, P. Tribedy and R. Venugopalan, Phys. Rev. Lett. 108, 252301 (2012) doi:10.1103/PhysRevLett.108.252301 [arXiv:1202.6646 [nucl-th]].

[33] B. Schenke, P. Tribedy and R. Venugopalan, Phys. Rev. C 86, 034908 (2012) doi:10.1103/PhysRevC.86.034908 [arXiv:1206.6805 [hep-ph]].

[34] J. S. Moreland, J. E. Bernhard and S. A. Bass, Phys. Rev. C 92, no. 1, 011901 (2015) doi:10.1103/PhysRevC.92.011901 [arXiv:1412.4708 [nucl-th]]. 